\title{
Injective endomorphisms of algebraic and analytic sets
}

\author{
by SŁAwOmir Cynk and Kamil Rusek (Kraków)
}

\begin{abstract}
We prove that every injective endomorphism of an affine algebraic variety over an algebraically closed field of characteristic zero is an automorphism. We also construct an analytic curve in $\mathbb{C}^{6}$ and its holomorphic bijection which is not a biholomorphism.
\end{abstract}

Introduction. The Zariski Main Theorem (see e.g. [4]) asserts that every injective morphism of normal algebraic varieties over an algebraically closed field of characteristic zero is an automorphism onto its open image. For arbitrary complex algebraic varieties regularity of the inverse of a regular biholomorphic bijection is ensured by the Serre Theorem [7, 9]. The simple example $\mathbb{C} \ni t \rightarrow\left(t^{2}, t^{3}\right) \in\left\{x^{3}-y^{2}=0\right\}$ shows that in general, bijective morphisms are not isomorphisms. From the example $\{x y-1=0\} \ni(u, v) \rightarrow$ $(u, 0) \in\{y=0\}$ we see that an injective morphism of non-singular algebraic sets may not be surjective.

Grothendieck proved in [5, Prop. 17.9.6] a counterpart of the Zariski Main Theorem for the category of S-preschemes, where $\mathrm{S}$ is a fixed prescheme. His theorem says that every injective S-endomorphism of an Sprescheme of finite presentation is an automorphism. Note that his theorem is unapplicable to endomorphisms of algebraic sets.

Our main result gives a geometric counterpart of the Grothendieck result; Theorem 2.2 says that every injective endomorphism of an affine algebraic variety over an algebraically closed field $\mathbf{k}$ of characteristic zero is an automorphism. This generalizes the well-known result of Białynicki-Birula and Rosenlicht [2] saying that every injective polynomial transformation of $\mathbf{k}^{n}$ is a polynomial automorphism.

Note that surjectivity of an injective endomorphism of an affine algebraic variety was earlier proved by $\mathrm{Ax}[1]$. In the proof $\mathrm{Ax}$ applied the so-called $32 \mathrm{C} 25$.

1991 Mathematics Subject Classification: Primary: 14A10, 32C15; Secondary 14E99, 
"transfer principle" and the metamathematical notion of an "elementary formula". A topological proof of Ax's Theorem was given by Borel in his unpublished paper [3].

One can also consider Theorem 2.2 as an analogue of the ClementsOsgood Theorem on analyticity of the inverse of a holomorphic bijection of a complex manifold. In Section 3 we show that there is no counterpart of Theorem 2.2 for the category of analytic sets. In order to obtain a suitable counterexample we construct an irreducible one-dimensional complex analytic space and its holomorphic but not biholomorphic bijection and next we prove that this analytic space is biholomorphic with an analytic curve in $\mathbb{C}^{6}$.

1. Dominating regular mappings with finite fibres. Let $F: V \rightarrow$ $W$ be a regular dominating mapping of irreducible affine algebraic varieties over k, i.e. $\overline{F(V)}=W$. Then the induced homomorphism $F_{*}: \mathbf{k}[W] \rightarrow \mathbf{k}[V]$ of rings of regular functions is injective, so it has a unique extension to a monomorphism of fields of rational functions, also denoted by $F_{*}: \mathbf{k}(W) \rightarrow$ $\mathbf{k}(V)$. If we assume additionally that $\operatorname{dim} V=\operatorname{dim} W$ then the field $\mathbf{k}(V)$ is a finite extension of the field $F_{*}(\mathbf{k}(W))$; we put $d(F)=\left[\mathbf{k}(V): F_{*}(\mathbf{k}(W))\right]$. It is well known that $d(F)$ is equal to the number of points in the generic fibre of $F$ (see e.g. [4]).

In some cases we have more precise information about the number $d(F)$ :

THEOREM 1.1. Let $V$ and $W$ be normal affine varieties and $\operatorname{dim} V=$ $\operatorname{dim} W$. If $F: V \rightarrow W$ is a regular dominating mapping with finite fibres then $d(F)=\max \left\{\# F^{-1}(y): y \in W\right\}$.

For the proof we need the following

Lemma 1.2. Let $V$ and $W$ be irreducible affine algebraic varieties such that $\operatorname{dim} V=\operatorname{dim} W$ and $V$ is normal. Assume that $F: V \rightarrow W$ is a regular dominating mapping with finite fibres. Then, for every $y \in W$ with $F^{-1}(y)=\left\{x_{1}, \ldots, x_{m}\right\} \subset V$, there exists a function $f \in \mathbf{k}[V]$ which is integral over $F_{*}(\mathbf{k}[W])$ and $f\left(x_{i}\right) \neq f\left(x_{j}\right)$ for $i \neq j$.

P r o of. By [4, Corollary 1, p. 136] we can find a normal affine algebraic variety $W^{\prime}$, a regular injective mapping $F^{\prime}: V \rightarrow W^{\prime}$ and a regular finite mapping $G: W^{\prime} \rightarrow W$ such that $F=G \circ F^{\prime}$.

Then the points $\left\{F^{\prime}\left(x_{j}\right): j=1, \ldots, m\right\} \subset W^{\prime}$ are pairwise different, so there exists $g \in \mathbf{k}\left[W^{\prime}\right]$ such that $g\left(F^{\prime}\left(x_{i}\right)\right) \neq g\left(F^{\prime}\left(x_{j}\right)\right)$ for $i \neq j$. Since $G$ is finite, the ring $\mathbf{k}\left[W^{\prime}\right]$ is integral over the ring $G_{*}(\mathbf{k}[W])$. Therefore $f=g \circ F^{\prime}=F_{*}^{\prime}(g)$ has all desired properties.

Proof of Theorem 1.1. Take a $y \in W$ and the function $f \in \mathbf{k}[V]$ from Lemma 1.2 which is integral over the ring $F_{*}(\mathbf{k}[W])$ and separates the 
points in the fibre $F^{-1}(y)=\left\{x_{1}, \ldots, x_{m}\right\} \subset V$.

In the extension of integral rings $F_{*}(\mathbf{k}[W]) \subset \mathbf{k}[V]$ the ring $F_{*}(\mathbf{k}[W])$ is normal. One can easily verify that $f \in \mathbf{k}[V]$ is algebraic over the field $F_{*}(\mathbf{k}(W))$ and the coefficients of its minimal equation of degree $d$ over $F_{*}(\mathbf{k}(W))$ lie in $F_{*}(\mathbf{k}[W])$. Since all functions from $F_{*}(\mathbf{k}[W])$ are constant on the set $F^{-1}(y)$ whose elements are separated by $f$, we have $m \leq d \leq d(F)$. Hence $d(F)=\max \left\{\# F^{-1}(y): y \in W\right\}$.

2. Bijectivity implies biregularity. Assume that all varieties occurring in this section are defined over a field $\mathbf{k}$ (algebraically closed of characteristic zero).

Lemma 2.1. Let $V$ be an affine algebraic variety and let $V=V_{1} \cup \ldots \cup V_{s}$ be its decomposition into irreducible components. Denote by $\pi_{i}: \widehat{V}_{i} \rightarrow V_{i}$ a normalization of $V_{i}$ for $i=1, \ldots, s$. Then

$$
M_{V}=\left\{f: V \rightarrow \mathbf{k}: f \circ \pi_{i} \in \mathbf{k}\left[\widehat{V}_{i}\right] \quad \text { for } i=1, \ldots, s\right\}
$$

is a noetherian $\mathbf{k}[V]$-module (under the standard operations).

Proof. Since $\mathbf{k}\left[\widehat{V}_{i}\right]$ is a noetherian $\mathbf{k}\left[V_{i}\right]$-module, it is also a noetherian $\mathbf{k}[V]$-module for every $i=1, \ldots, s$ (under the multiplication $r f=\left(r \circ \pi_{i}\right) f$ for $r \in \mathbf{k}[V], f \in \mathbf{k}\left[\widehat{V}_{i}\right]$.) The mapping

$$
M_{V} \ni f \rightarrow\left(f \circ \pi_{1}, \ldots, f \circ \pi_{s}\right) \in \bigoplus_{i=1}^{s} \mathbf{k}\left[\widehat{V}_{i}\right]
$$

is a monomorphism of $\mathbf{k}[V]$-modules. Therefore $M_{V}$ is a noetherian $\mathbf{k}[V]$ module.

Let $V$ be an irreducible affine algebraic variety over $\mathbf{k}$ and let $\widehat{V}$ together with a finite surjective regular and birational mapping $\pi: \widehat{V} \rightarrow V$ be a normalization of $V$. By the universal property of normalization, for a given regular endomorphism $F: V \rightarrow V$ there exists a unique endomorphism $\widehat{F}: \widehat{V} \rightarrow \widehat{V}$ such that $\pi \circ \widehat{F}=F \circ \pi$. We have

Lemma 2.2. (i) If $F$ is injective then $\widehat{F}$ is injective.

(ii) If $\widehat{F}$ is surjective then $F$ is surjective.

Proof. (i) Assume that $F$ is injective. First observe that $\widehat{F}$ is dominating. Since $F$ is dominating and $\pi: \widehat{V} \rightarrow \widehat{V}$ is surjective, we see that $F \circ \pi: \widehat{V} \rightarrow V$ is dominating. Since $\pi$ is closed, $\pi(\overline{\widehat{F}(\widehat{V})})=\overline{\pi(\widehat{F}(\widehat{V}))}=$ $\overline{F(\pi(\widehat{V}))}=V$. If $\widehat{\widehat{F}(\widehat{V})} \varsubsetneqq \widehat{V}$ then by irreducibility of $\widehat{V}$ we would have $\operatorname{dim} V=\operatorname{dim} \pi(\widehat{\widehat{F}(\widehat{V})}) \leq \operatorname{dim} \overline{\widehat{F}(\widehat{V})}<\operatorname{dim} \widehat{V}$, a contradiction. 
Therefore $\overline{\widehat{F}(\widehat{V})}=\widehat{V}$. Moreover, $1=d(F) d(\pi)=d(F \circ \pi)=d(\pi \circ \widehat{F})=$ $d(\widehat{F})$. Since all fibres of $\pi$ are finite, the same holds for $\widehat{F}$. By Theorem 1.1, $\left\{y \in \widehat{V}: \# \widehat{F}^{-1}(y) \neq 1\right\}=\left\{y \in \widehat{V}: \widehat{F}^{-1}(y)=\emptyset\right\}$, which means that $\widehat{F}$ is injective.

(ii) If $\widehat{F}$ is surjective then $\pi \circ \widehat{F}=F \circ \pi$ is also surjective, and hence so is $F$.

Our main result is

THEOREM 2.2. Let $V$ be an affine algebraic variety and let $F: V \rightarrow V$ be a regular mapping. Then the following statements are equivalent:

(i) $F$ is injective.

(ii) $F$ is a bijection of $V$.

(iii) $F$ is an automorphism of $V$.

Proof. The implication (i) $\Rightarrow$ (ii) is true by the Ax Theorem and (iii) implies (i).

(ii) $\Rightarrow$ (iii). Assume that $V$ is an algebraic subset of $\mathbf{k}^{n}$ for some $n \in \mathbb{N}$ and $V=V_{1} \cup \ldots \cup V_{s}$ is the decomposition of $V$ into irreducible components. Denote by $\pi_{i}: \widehat{V}_{i} \rightarrow V_{i}$ a normalization of $V_{i}$ for $i=1, \ldots, s$. Then, by Lemma 2.1,

$$
\begin{aligned}
M_{V}^{n}=\bigoplus_{1}^{n} M_{V}=\{G: V \rightarrow & \mathbf{k}^{n}: \\
& \left.\quad G \circ \pi_{i}: \widehat{V}_{i} \rightarrow \mathbf{k}^{n} \text { is regular for } i=1, \ldots, s\right\}
\end{aligned}
$$

is a noetherian $\mathbf{k}[V]$-module.

Without loss of generality we can assume that $F\left(V_{i}\right) \subset V_{i}$ for $i=1, \ldots, s$. Let $F_{i}$ denote the restriction of $F$ to $V_{i}$. By Lemma 2.2, the Ax Theorem and Zariski's Main Theorem, every mapping $\widehat{F}_{i}$ is an automorphism of $\widehat{V}_{i}$. Therefore, for every $\nu \in \mathbb{N}$ and every $i \in\{1, \ldots, s\}$, the mapping $F^{-\nu} \circ \pi_{i}=$ $\pi_{i} \circ\left(\widehat{F}_{i}\right)^{-\nu}$ is regular, so $F^{-\nu} \in M_{V}^{n}$.

For every $m \in \mathbb{N}$ we put $S_{m}=\mathbf{k}[V] F^{-1}+\ldots+\mathbf{k}[V] F^{-m}$. Then the $S_{m}$ are submodules of the noetherian $\mathbf{k}[V]$-module $M_{V}^{n}$ and $S_{1} \subset S_{2} \subset S_{3} \subset \ldots$ Therefore there exists an $l \in \mathbb{N}$ such that $S_{l}=S_{l+1}$ and we can write

$$
F^{-(l+1)}=\sum_{j=1}^{l} r_{j} F^{-j} \quad \text { for some } r_{1}, \ldots, r_{l} \in \mathbf{k}[V] .
$$

Thus the mapping

$$
F^{-1}=F^{-(l+1)} \circ F^{l}=\sum_{j=1}^{l}\left(r_{j} \circ F^{l}\right) F^{l-j}
$$

is regular and the proof is complete. 
Remark. Note that in [8] Matsumura and Monsky gave examples of hypersurfaces in $\mathbb{C}^{n}$ having no automorphisms (hence no regular injective endomorphisms) apart from the identity.

3. Counterexample in the analytic case. We first construct an analytic space and a holomorphic bijection of this space onto itself which is not a biholomorphism.

ExAMPLE 3.1. Let $X$ denote the topological space obtained from $Y=$ $\mathbb{C} \backslash(\mathbb{Z} \backslash \mathbb{N})$ by gluing every pair of points $l$ and $l+1 / 2$ into one point $a_{l} \in X$ for $l \in \mathbb{N}$. Let $\pi: Y \rightarrow X$ denote the canonical mapping defining this quotient Hausdorff space.

We have $X=X_{0} \cup\left\{a_{0}, a_{1}, \ldots\right\}$, where $X_{0}=\mathbb{C} \backslash(\mathbb{Z} \cup\{k+1 / 2: k \in \mathbb{N}\})$ is an open subset of $X$. Moreover,

$$
U_{l}=\left\{a_{l}\right\} \cup(B(l, 1 / 5) \backslash\{l\}) \cup(B(l+1 / 2,1 / 5) \backslash\{l+1 / 2\})
$$

is a neighbourhood of $a_{l}$ in $X .(B(z, r)$ denotes the open ball with centre $z \in \mathbb{C}$ and radius $r>0$.)

We define a complex structure on $X$ by the family of homeomorphisms:

$$
\begin{aligned}
& \operatorname{id}_{X_{0}}: X_{0} \rightarrow X_{0}, \\
& \phi_{l}: U_{l} \rightarrow(B(0,1 / 5) \times\{0\}) \cup(\{0\} \times B(0,1 / 5)), \quad l \in \mathbb{N},
\end{aligned}
$$

where

$$
\phi_{l}(z)= \begin{cases}0 & \text { if } z=a_{l} \\ (z-l, 0) & \text { if } z \in B(l, 1 / 5) \backslash\{l\} \\ (0, z-l-1 / 2) & \text { if } z \in B(l+1 / 2,1 / 5) \backslash\{l+1 / 2\} .\end{cases}
$$

They define on $X$ the structure of a one-dimensional irreducible complex space with singularities exactly at the points $a_{l}$; we write $\operatorname{Sing} X=\left\{a_{l}\right.$ : $l \in \mathbb{N}\}$.

Define the mapping $\Phi: X \rightarrow X$ by the formula

$$
\Phi(x)= \begin{cases}x+1 & \text { if } x \in X_{0} \backslash\{-1 / 2\} \\ a_{l+1} & \text { if } x=a_{l}, l \in \mathbb{N} \\ a_{0}, & \text { if } x=-1 / 2\end{cases}
$$

Then $\Phi$ is a holomorphic bijection of $X$ onto $X$ but not an open mapping (it is not open at the point $-1 / 2$ ). Therefore $\Phi^{-1}: X \rightarrow X$ is not holomorphic.

The complex space $X$ constructed above can be "well" embedded in $\mathbb{C}^{6}$ :

Proposition 3.2. There exists a biholomorphic mapping of $X$ onto an irreducible analytic curve $V \subset \mathbb{C}^{6}$. 
Pr o of. Since $X$ is one-dimensional, it is a Stein space (see $[6$, Theorem IX.B.10]). Therefore there exists a holomorphic homeomorphism $\theta$ of $X$ onto an irreducible analytic curve in $\mathbb{C}^{4}$ (see [6, Theorem VII.C.10]).

Observe that the set $\left\{x \in X: \operatorname{rank}_{x} \theta<\operatorname{dimt}_{x} X\right\}$ is contained in $\pi\left(\left\{x \in X_{0}: d_{x}(\theta \circ \pi)=0\right\} \cup \operatorname{Sing} X\right.$, so it is at most countable. (Following [6], $\operatorname{dimt}_{x} X$ denotes the tangential dimension of $X$ at $x ; \operatorname{rank}_{x} \theta$ denotes the rank of the induced linear mapping of Zariski tangent spaces.)

Since $\operatorname{dimt}_{x} X \leq 2$ for every $x \in X$, applying at most twice Lemma VII.C.11 of [6] we can find a holomorphic homeomorphism $\Psi$ of $X$ onto an irreducible analytic curve $V$ in $\mathbb{C}^{6}$ such that $\operatorname{rank}_{x} \Psi=\operatorname{dimt}_{x} X$ for every $x \in X$.

We claim that $\Psi$ is a biholomorphism of $X$ onto $V$. Indeed, take an $x_{0} \in X$ and a complex manifold $M$ of dimension $\operatorname{dimt}_{x_{0}} X$ such that $M \cap X$ is a neighbourhood of $x_{0}$ in $X$. Let $\widetilde{\Psi}$ be a holomorphic extension of $\Psi$ to $M$. Then $\operatorname{rank}_{x_{0}} \widetilde{\Psi}=\operatorname{dimt}_{x_{0}} X$. Since the function $M \ni x \rightarrow \operatorname{rank}_{x} \widetilde{\Psi} \in \mathbb{N}$ is lower semicontinuous, shrinking $M$ if necessary we conclude by the constant rank theorem that $\widetilde{\Psi}: M \rightarrow \mathbb{C}^{6}$ is a biholomorphism onto its image $N=\widetilde{\Psi}(M)$. Then $\widetilde{\Psi}^{-1}(N \cap \Psi(X))=M \cap X$, i.e. $\widetilde{\Psi}^{-1}=\Psi^{-1}$ in a neighbourhood of $\Psi\left(x_{0}\right)$ in $V=\Psi(X)$. Therefore $\Psi^{-1}$ is holomorphic at $\Psi\left(x_{0}\right) \in V$, and this proves that $\Psi$ is a biholomorphic mapping of $X$ onto $V$, as desired.

The above constructions give

EXAMPLE 3.3. The mapping $F=\Psi \circ \theta \circ \Psi^{-1}: V \rightarrow V$ is a holomorphic homeomorphic bijection of the irreducible analytic curve $V \subset \mathbb{C}^{6}$ onto itself with $F^{-1}$ not holomorphic.

In this context the following question seems to be interesting:

PRoBlem. Is each holomorphic bijective self-transformation of an algebraic set necessarily biholomorphic?

\section{References}

[1] J. Ax, A metamathematical approach to some problems in number theory, in: Proc. Sympos. Pure Math. 20, Amer. Math. Soc., 1971, 161-190.

[2] A. Białynicki-Birula and M. Rosenlicht, Injective morphisms of real algebraic varieties, Proc. Amer. Math. Soc. 13 (1962), 200-203.

[3] A. Borel, Injective endomorphisms of algebraic varieties, preprint.

[4] J. Dieudonné, Cours de géométrie algébrique, Vol. II, Presses Univ. France, 1974.

[5] A. Grothendieck, Eléments de géométrie algébrique. IV. Etude locale des schémas et des morphismes de schémas (quatrième partie), Inst. Hautes Etudes Sci. Publ. Math. 32 (1967).

[6] R. C. Gunning and H. Rossi, Analytic Functions of Several Complex Variables, Prentice-Hall, 1965. 
[7] S. Łojasiewicz, An Introduction to Complex Analytic Geometry, PWN, Warszawa 1988 (in Polish).

[8] H. Matsumura and P. Monsky, On the automorphisms of hypersurfaces, J. Math. Kyoto Univ. 3 (3) (1964), 347-361.

[9] J.-P. Serre, Géométrie algébrique et géométrie analytique, Ann. Inst. Fourier (Grenoble) 6 (1955-56), 1-42.

INSTITUTE OF MATHEMATICS

JAGIELLONIAN UNIVERSITY

REYMONTA 4

30-059 KRAKÓW, POLAND

Reçu par la Rédaction le 2.11.1989

Révisé le 20.3.1991 